\title{
多维线性汇流系统的识别与长办 汇流曲线参数的计算公式
}

\author{
李心铭罗钟锍 \\ （水电部长江流域规划办公室水文局，武汉）
}

\section{一、多维线性定常汇流系统}

考虑净雨空间分布不均, 运用河槽汇流曲线进行以等流时块为单元区的流域汇流推算或 以支流流域为单元区的河网汇流演算，以及考虑区间支流的河道演算等问题，虽然情况各异， 但具有共同的特点: 一是人流非单一;二是出流由各人流通过各自相应流程的河槽汇流曲线，

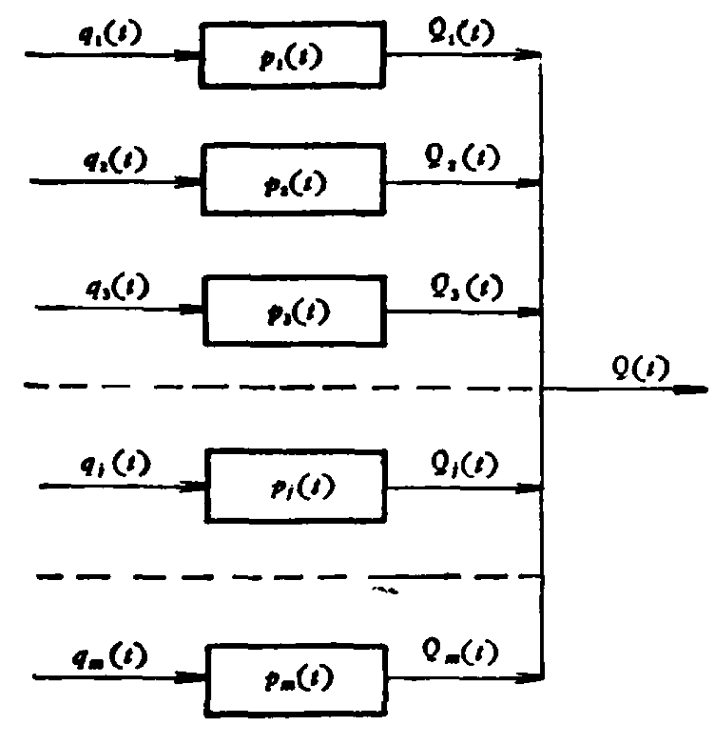

因 1 多维线性定常汇流系统框图

转换得的单元出流过程叠加而得. 因此为便 于问题的研究，将这几种汇流问题归纳为多 点入流汇流问题，而把单位线方法以及总入 流河道演进法称为单点人流汇流问题. 根据 系统分析观点，这种汇流问题是多维线性定 常系统问题,系统框图如图 1 所示.

输人（净雨或入流流量过程）为连续型 时, 第 $i$ 子系统的输出 (单元出流过程)为

$$
Q_{i}(t)-\int_{0}^{t} q_{i}(\xi) p_{i}(t-\xi) d \xi,
$$

输人为阶梯型时

$$
Q_{i}(t)=\sum_{i=1}^{r_{j}} \bar{q}_{i i} P_{i}(t-(i-1) \Delta t) .
$$

又系统输出(出流过程)为 $Q(t)-\sum_{j=1}^{m} Q_{j}(t)$, 因此其多维线性定常汇流系统方程分别为

$$
\begin{gathered}
Q(t)-\sum_{i=1}^{m} \int_{0}^{t} q_{i}(\xi) p_{j}(t-\xi) d \xi, \\
Q(t)-\sum_{j=1}^{m} \sum_{i=1}^{i_{i}} \bar{q}_{i i} P_{i}(t-(i-1) \Delta t),(t-(i-1) \Delta t \geqslant 0),
\end{gathered}
$$

式中 $t$ 与 $\Delta t$ 分别为时程与梯型输人的单位时段、汇流曲线的人流历时或计算时段; $m$ 为子系 统数(等流时块数、河网或河道的人流数); $q$ 与 $\bar{q}$ 分别为连续型与阶梯型输人(等流时块上的 净雨, 河网或河道的入流); $P$ 与 $P$ 为子系统的瞬时与时段两种情况的传递函数(瞬时与时段汇 流曲线); $r$ 为阶梯型输人情况的人流时段数; $Q_{j}$ 与 $Q$ 为第 $i$ 子系统的输出与系统输出(第 $i$

本文1984年11月9日收到. 
单元出流与出流).

从系统方程可以看出,每个子系统的汇流曲线不同,这就反应了此方法,用于河道汇流时， 考虑了干流上游断面和区间各支流人流点至下游断面的距离不等应受的不同调节作用; 用于 流域或河网汇流时,考虑了流域上不同远近的来水受到不同的调节作用，因而比将流域上呈现 为空间分布状态的净雨过程简化成单一净雨过程的单位线类方法, 以及干支流入.流综合成一 总人流的河道演算法的概念合理.

\section{二、系统的识别}

从汇流系统方程知,系统输人已知, 当汇流曲线确定后, 即可算出系统输出. 显然关键在 于汇流曲线参数的定量. 解决此问题的思路是首先推导出系统中各子系统的人流、相应的汇 流曲线、出流三者的矩关系式, 以及汇流曲线的矩与汇流参数的关系式, 然后通过此两关系式 的联解,得出汇流参数的计算公式.

\section{1. 入流过程为连续型}

1. 出流与各人流及其相应的瞬时汇流曲线三者的一阶原点矩关系式: 直接根据式 (3) 推 导矩关系式颇烦，为此由卷积定理将它变换为频域表达的象函方程:

$$
\widetilde{Q}(s)=\sum_{j=1}^{m} \tilde{p}_{i}(s) \tilde{q}_{i}(s),
$$

式中 $\widetilde{Q}(s)-L[Q(t)] ; \tilde{p}_{i}(s)=L\left[p_{i}(t)\right] ; \tilde{q}_{i}(s)=L\left[q_{i}(t)\right]$.

微分式(5)并令 $s=0$ 得

$$
\left.\frac{d \widetilde{Q}(s)}{d s}\right|_{s=0}-\left.\sum_{j=1}^{m} \tilde{p}_{j}(s) \frac{d \tilde{q}_{j}(s)}{d s}\right|_{s=0}+\left.\sum_{j=1}^{m} \tilde{q}_{i}(s) \frac{d \tilde{p}_{i}(s)}{d s}\right|_{s=0} .
$$

根据拉氏变换微分定理与一阶原点矩定义有

$$
\begin{gathered}
\left.\frac{d}{d s} \tilde{Q}(s)\right|_{s=0}=-T_{Q} \int_{0}^{\infty} Q(t) d t ;\left.\frac{d}{d s} \tilde{q}_{i}(s)\right|_{s=0}=-T_{q_{j}} \int_{0}^{\infty} q_{j}(t) d t ; \\
\left.\frac{d}{d s} \tilde{p}_{i}(s)\right|_{s=0}=-T_{p_{i}} \int_{0}^{\infty} p_{j}(t) d t ;\left.\tilde{q}_{j}(s)\right|_{s=0}-\int_{0}^{\infty} q_{j}(t) d t ; \\
\left.\tilde{p}_{i}(s)\right|_{s=0}-\int_{0}^{\infty} p_{j}(t) d t=1,
\end{gathered}
$$

并令 $\omega_{i}-\int_{0}^{\infty} q_{j}(t) d t / \int_{0}^{\infty} Q(t) d t$ 即得 $T_{Q}=\sum_{j=1}^{m} \omega_{i} T_{q_{j}}+\sum_{j=1}^{m} \omega_{i} T_{p_{j}}$,

式中 $T_{Q}, T_{q_{j}} 、 T_{p_{j}}$ 为 $Q(t) ， q_{j}(t) 、 p_{j}(t)$ 的一阶原点矩; $\omega_{j}$ 为第 $i$ 人流的相对水量.

2. 出流二阶原点矩与每个人流及其相应的瞬时汇流曲线的一、二阶原点矩关系式: 对式 (5)进行两次微分并令 $s=0$ 得

$$
\begin{aligned}
\left.\frac{d^{2}}{d s^{2}} \tilde{Q}(s)\right|_{s=0}= & \left.\sum_{j=1}^{m} \tilde{p}_{i}(s) \frac{d^{2}}{d s^{2}} \tilde{q}_{i}(s)\right|_{s=0}+\left.\sum_{j=1}^{m} \tilde{q}_{i}(s) \frac{d^{2}}{d s^{2}} \tilde{p}_{i}(s)\right|_{s=0} \\
& +\left.2 \sum_{j=1}^{m} \frac{d}{d s} \tilde{q}_{i}(s) \frac{d}{d s} \tilde{p}_{i}(s)\right|_{s=0},
\end{aligned}
$$

因 


$$
\begin{gathered}
\left.\frac{d^{2}}{d s^{2}} \tilde{q}_{i}(s)\right|_{,=0}=v_{q_{i}} \int_{0}^{\infty} q_{i}(t) d t ;\left.\frac{d^{2}}{d s^{2}} \tilde{p}_{i}(s)\right|_{,=0}=v_{p_{j}} ; \\
\left.\frac{d^{2}}{d s^{2}} \widetilde{Q}(s)\right|_{s=0}=v_{Q} \int_{0}^{\infty} Q(t) d t,
\end{gathered}
$$

故

$$
\nu_{Q}=\sum_{j=1}^{m} \omega_{j} \nu_{q_{j}}+\sum_{j=1}^{m} \omega_{j} \nu_{p_{j}}+2 \sum_{j=1}^{m} \omega_{i} T_{q_{j}} T_{p_{i}},
$$

式中 $v_{Q}$ 为 $Q(t)$ 的二阶原点矩; $v_{q_{j}} 、 v_{p_{j}}$ 为 $q_{i}(t) 、 p_{j}(t)$ 的二阶原点矩.

\section{2. 人法过程为胟梯珰}

众所周知,历时为 $\Delta t$ 的矩形入流的一阶原点矩为 $\Delta t / 2$, 因此它与瞬时汇流曲线及其出 流三者的一阶原点矩的关系有

$$
T_{p_{j}}=T_{p_{i}}-\frac{\Delta t}{2},
$$

将式(10)代人式(7) 即得阶梯型入流情况， $Q(t) 、 q(t) 、 P(t)$ 三者的一阶原点矩关系式如下:

$$
T_{Q}=\sum_{j=1}^{m} \omega_{j} T_{q_{j}}+\sum_{j=1}^{m} \omega_{j} T_{P_{j}}-\frac{\Delta t}{2} .
$$

又知历时 $\Delta t$ 的矩形入流的二阶中心矩为 $\frac{\Delta t^{2}}{12}$, 因此它与瞬时汇流曲线及其出流三者的 二阶中心矩的关系为

$$
\mu_{p_{j}}=\mu_{P_{i}}-\frac{\Delta t^{2}}{12}
$$

再者,由统计数学知,

$$
\mu_{p_{i}}=\nu_{p_{i}}-T_{p i}^{2} ; \mu_{P_{i}}=\nu_{p_{i}}-T_{P_{i}}^{2},
$$

将式(13)代人式(12),而后代人式(10)得

$$
\nu_{p_{i}}=\nu_{p_{j}}-\Delta t T_{P_{i}}+\Delta \iota^{2} / 6,
$$

式(14)代人式(9)即得梯型入流情况下 $Q(t)$ 的二阶原点矩与 $\bar{q}(t) 、 P(t)$ 的一、三阶原点矩 关系式:

$$
\nu_{Q}=\sum_{j=1}^{m} \omega_{j}\left(v_{q_{j}}-T_{q_{j}} \Delta t\right)+\sum_{j=1}^{m} \omega_{i}\left(\nu_{P_{i}}-\Delta t T_{P_{j}}\right)+2 \sum_{j=1}^{m} \omega_{j} T_{P_{j}} T_{q_{j}}+\frac{\Delta t^{2}}{6},
$$

式中 $\mu_{P} 、 \mu_{P}$ 与 $T_{P} 、 \nu_{P}$ 为 $P(t) 、 P(t)$ 的二阶中心矩与 $P(t)$ 的一、二阶原点矩.

\section{三、多维线性定常汇流系统中长办汇流曲线的参数计算公式}

\section{1. 长办汇流曲线的矩与汇流参数关系式}

长办汇流曲线是笔者于 1962 年,在 $\Gamma . \Pi$. 加里宁 ${ }^{[2]}$ 河槽汇流曲线的概念基础上研究出的 一种人流为矩形的河槽汇流曲线,或称时段河槽汇流曲线,其含义是: 一历时为 $t_{K}$ 、流量为 1

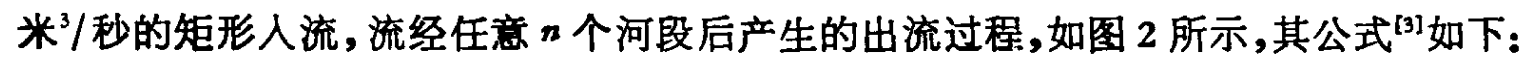

$$
P_{n}= \begin{cases}1-e^{-m} \sum_{i=0}^{n-1} \frac{m^{i}}{i !}, & \left(0 \leqslant m \leqslant m_{K}\right), \\ \sum_{j=0}^{n-1} \frac{M^{i}}{i !} e^{-M}\left(1-e^{-m_{K}} \sum_{i=0}^{m-i-1} \frac{m_{K}^{i}}{i !}\right), & \left(m_{K} \leqslant m\right),\end{cases}
$$


式中 $M=m-m_{K} ; m=\frac{t}{\tau} ; m_{K}=\frac{t_{K}}{\tau} ; n$ 与 $\tau$ 为流经河段数与汇流时间; $t_{K}$ 为人流历时; $P_{n}$ 为河段数为 $n$ 的长办汇流曲线.

长办汇流曲线的任意 $Z$ 阶原点矩为

$$
\begin{aligned}
M_{Z}= & \left\{\int_{0}^{m_{K}} m^{z}\left(1-e^{-m} \sum_{i=0}^{n-1} \frac{m^{i}}{i !}\right) d_{m}\right. \\
& +\int_{0}^{\infty}\left(m_{K}+M\right)^{z}\left[\sum_{j=0}^{n-1} \frac{M^{i}}{j !} e^{-M}\right. \\
& \left.\left.\cdot\left(1-e^{-m_{K}} \sum_{i=0}^{n-i-1} \frac{m_{K}^{i}}{i !}\right)\right] d M\right\} / m_{K},
\end{aligned}
$$

当 $Z=1$ 与 2 时,积分之,并考虑

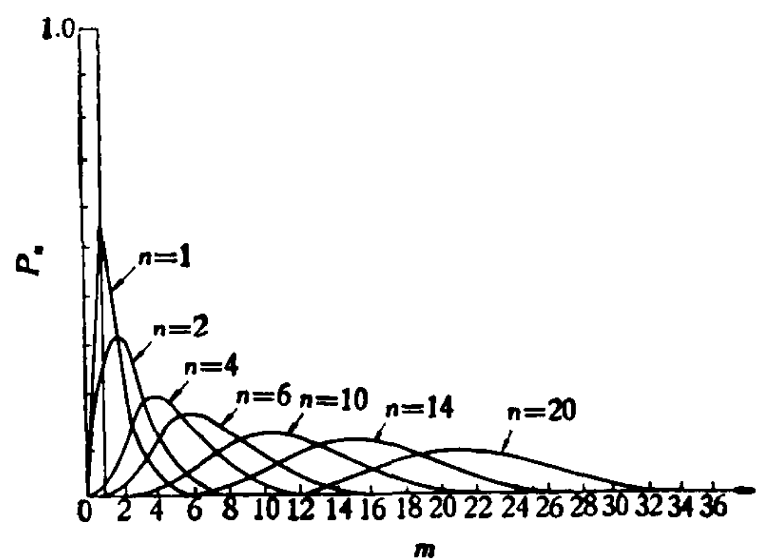

图 2 长办汇流曲线 $\left(m_{K}=1\right.$ 情况 $)$

$$
m_{K}=\frac{t_{K}}{\tau}, M_{1}=T_{P} / \tau, M_{2}=\nu_{P} / \tau^{2},
$$

则得长办汇流曲线的一、二阶原点矩与汇流参数 $n 、 \tau$ 的关系式为

$$
\begin{gathered}
T_{P}=n \tau+t_{K} / 2, \\
\nu_{P}=n(n+1) \tau^{2}+n \tau t_{K}+t_{K}^{2} / 3 .
\end{gathered}
$$

\section{2. 汇流数 $n 、 \tau$ 的计算公式}

长办汇流曲线是矩形人流的汇流曲线,适用于阶梯型入流情况,因此应联解式 (11)、(15) 与式(18)、(19), 推导 $n 、 \tau$ 的计算公式.

1. 区间有支流加人的河道或河网汇流情况: 设河网有 $m$ 条支流, 或演算河段的区间有 $m-1$ 条支流; 河网最远入流至流域出口或河道上断面至下断面的距离为 $l$ 与总河段数 $n$; 第 $i$ 人流至流域出口或下断面的距离为 $l_{i}$ 与河段数 $n_{i}$. 则

$$
n_{i}=n l_{j} / l=n L_{i},
$$

并令计算时段 $\Delta t=t_{K}$, 将式(18)、(20)代人式(11)得

$$
n \tau=\left(T_{Q}-\sum_{j=1}^{m} \omega_{j} T_{q_{i}}\right) / \sum_{j=1}^{m} \omega_{j} L_{i} .
$$

又将式(18)、(19)、(20)代入式(15)得

$$
\left(\sum_{j=1}^{m} L_{j}^{2} \omega_{j}\right) n^{2} \tau^{2}+\left(\sum_{j=1}^{m} \omega_{i} L_{j}\right) n \tau^{2}+2\left(\sum_{j=1}^{m} \omega_{j} L_{j} T_{q_{i}}\right) n \tau-v_{Q}-\sum_{j=1}^{m} \omega_{j} v_{q_{j} \bullet}
$$

联解式(21)与(22)即得汇流参数计算公式:

$$
\tau=\frac{c_{1}}{c_{0}}-\frac{2 c_{2} \sum_{j=1}^{m} \omega_{j} L_{i}+c_{0} \sum_{j=1}^{m} \omega_{j} L_{i}^{2}}{\left(\sum_{j=1}^{m} \omega_{j} L_{i}\right)^{2}}, n=c_{0} / \tau \sum_{j=1}^{m} \omega_{j} L_{i},
$$

其中

$$
c_{0}=T_{Q}-\sum_{j=1}^{m} \omega_{j} T_{q_{j}} ; \quad c_{1}=\nu_{Q}-\sum_{j=1}^{m} \omega_{j} \nu_{q_{j}} ; c_{2}=\sum_{j=1}^{m} \omega_{j} L_{i} T_{q_{j} \bullet}
$$

2. 等流时块为单元区的流域汇流情况: 设流域面积为 $A$, 平均雨深为 $R$, 等流时块数为 $n$; 第 $j$ 等流时块的面积为 $\Delta A_{i}$ 、净雨深 $R_{j}$. 当坡面汇流时间远远小于河道汇流时间而被 
略,则第 $i$ 河段的 $i$ 时段平均入流量为: $\bar{q}_{i_{i}}=R_{i_{i}} \Delta A_{j} / \Delta t$, 显然第 $j$ 等流时块的净雨一、二.阶 原点矩为

$$
T_{R_{j}}=T_{q_{j}}, v_{R_{i}}=v_{q_{j}}
$$

由于每个单元河段均有入流,因此 $m=n$, 则

$$
L_{i}=l_{i} / l=i / n,
$$

又

$$
\omega_{i}=\Delta A_{i} R_{j} / A R=f_{i} r_{i},
$$

将式(24)、(25)、(26)代人式(23), 即得等流时块为单元区情况的汇流参数计算公式:

$$
\tau=\frac{K_{1}}{K_{0}}-\frac{2 K_{2} \sum_{j=1}^{n} r_{i} f_{j} j+K_{0} \sum_{j=1}^{n} j^{2} r_{i} f_{i}}{\left(\sum_{i=1}^{n} j r_{i} f_{i}\right)^{2}}, \tau=K_{0} / \sum_{j=1}^{n} j r_{i} f_{j},
$$

其中

$$
K_{0}=T_{Q}-\sum_{j=1}^{n} f_{j} r_{j} T_{R_{j}} ; \quad K_{1}=v_{Q}-\sum_{j=1}^{n} f_{i} r_{i} \nu_{R_{i}} ; K_{2}=\sum_{j=1}^{n} i f_{i} r_{j} T_{R_{i}}
$$

\section{四、结 语}

1.著名的纳什单位线法,就系统观点而言,是一维线性定常系统, 因而是此法输入为 1 的 特例. 令式(7)、(9)与式(23)中的 $m=1$, 即可得纳什的净雨、洪水、瞬时单位线三者的矩关 系式与参数计算公式:

$$
\begin{gathered}
T_{Q}=T_{q}+T_{p}, \mu_{Q}=\mu_{q}+\mu_{p}, \tau=\left(\mu_{Q}-\mu_{q}\right) /\left(T_{Q}-T_{q}\right), \\
n=\left(T_{Q}-T_{q}\right)^{2} /\left(\mu_{Q}-\mu_{q}\right) .
\end{gathered}
$$

2. 长办汇流曲线公式的 $n$ 含有为使河段绳套蓖泄曲线单值化应采用的河段数的意义, 因 此应为正整数,然而,降雨和径流资料误差以及面雨深、基流分割、扣损和求矩等计算误差, 使 得计算的 $n$ 常非整数,这时应四舍五人取整. 同时必须注意保持 $n \tau$ 值不变对 $\tau$ 值进行修改.

3. 文中式(23)与(27)还可将瞬时汇流曲线的一、二阶原点矩与 $n, \tau$ 关系式, $T_{p_{i}}=n_{i} \tau$ 与 $\nu_{p_{j}}=n_{i} \tau^{2}+n_{i}^{2} \tau^{2}$ 代人式(7)与(9)推导得到.

[1] Nash., J. E., A unit hydrograph study with particular reference to British catchment, P. I. C. E., Vol. 17, 1960, 249-282.

[2] Калинин Г. П. и Милюков М. И., Придлижениый расqет неустановившегося движения водных масс, Tpydot L. Н. П., 6 (1958).

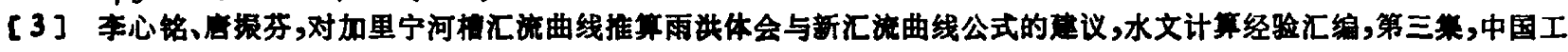
业出版社, 1965. 